\title{
Multispectral solutions in surveillance systems: the need for data fusion
}

\author{
M. Życzkowski, M. Szustakowski, W. Ciurapiński, M. Kastek, \\ R. Dulski, M. Karol, M. Kowalski \& P. Markowski \\ Institute of Optoelectronics, Military University of Technology, \\ Warsaw, Poland
}

\begin{abstract}
Surveillance systems are using more and more devices that use multiple spectral bands to detect threats, such as millimeter-wave radar, terahertz cameras, infrared cameras, and many others. These devices enable early detection of occurring threats. However, using such a wide spectrum of devices can significantly increase the amount of data collected by the system. This creates a need to determine the suitability of the information of individual devices, and reduce the amount of data. In this paper, the authors consider problems of data fusion from multispectral sensors and the possibilities of applications in security systems. The paper describes the opportunities and risks that potentially carry the use of data fusion, and shows the ability to detect and identify the target using data collected by multispectral sensors. A very important aspect of data fusion is to reduce the amount of data without losing contained information, and improve the readability, which facilitates its interpretation by a system operator. The paper focuses on determining the possible ranges of detection for selected multispectral devices capable of producing the correct synthesis of data. Keywords: surveillance system, laser photography, data fusion.
\end{abstract}

\section{Introduction}

Currently, there are many resources and materials which can easily be used for terrorist purposes. Organizations or single persons can cause significant damage for both critical infrastructure and civilians. Providing complex protection system is very difficult task due to many possible ways of performing the attacks. Specific kinds of risks detection due usually performed by dedicated 
sensing device. Increasing number of devices in entire system can overload operator console with too much information, making it difficult to initiate appropriate action. Threat detection is essential to carry out proper event verification and identification. Ensuring hazards identification for the entire area of large facilities is extremely difficult.

During projects our team is involved in hazard analysis, research in security technology and security systems designing for protection of key facilities.

Taking advantage of years of experience and latest technologies we developed integrated security systems of critical infrastructure that enables to precise detection and accurate identification of targets. During the research our team has developed a number of systems for critical infrastructure protection:

- Critical infrastructure integrated security system based on modules of underwater, above water and ground protection.

- Laser photography system for scene observation in limited visibility condition.

- THz system for dangerous objects detection.

Every single developed systems use multispectral detection to ensure the best detection performance. The systems are multispectral because they use sensors operating in the microwave, terahertz, infrared, and visible radiation bands. To optimize and improve readability of the operator interface in each of the systems common information method - data fusion was applied.

\section{Multispectral surveillance system}

Modern ground, surface and underwater monitoring systems, has a very high observation and measurement potential. These solutions play very important role in modern security systems.

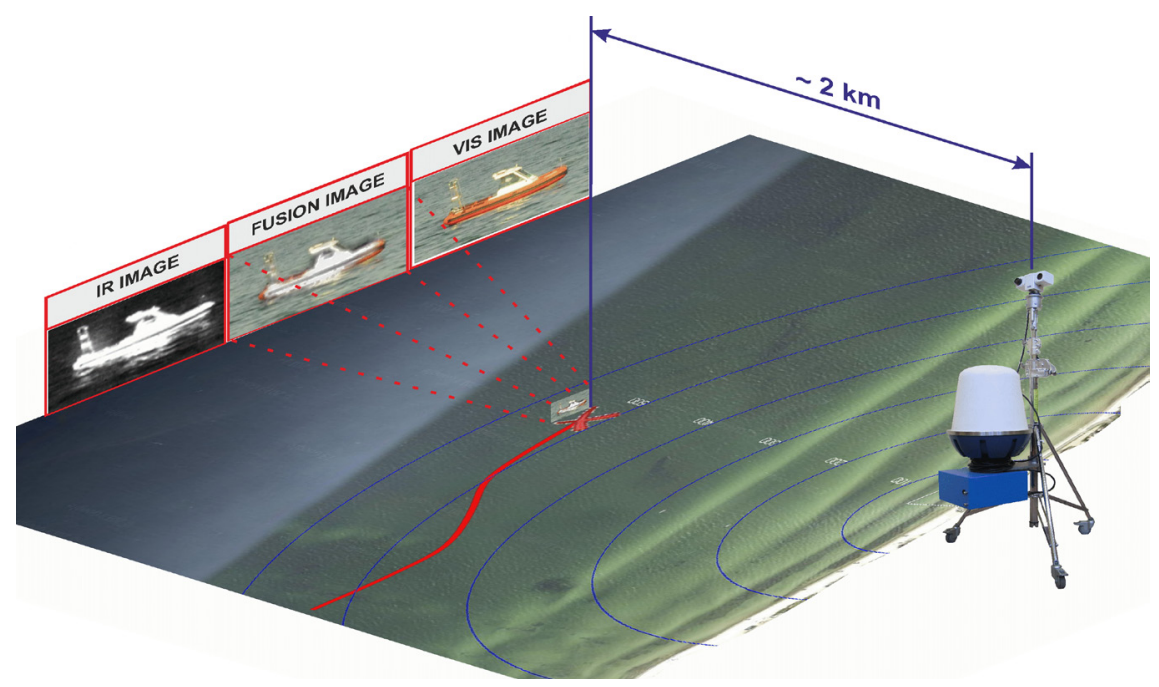

Figure 1: Integrated radar-camera mobile unit. 
An exemplary system of ground and above water perimeter protection has features like:

a) short range millimeter wave radars detecting small targets and flying objects to protect the ground and surface of water [1];

b) two coupled visualization technologies and identification of protected area scene - vision and thermal camera $[2,3]$;

c) Command and Control system managing alarms and transmitting video image of alarm to protection group.

Thermal and vision cameras, software coupled with radar ensure alarm visualization and identification in 24/7 regime. These systems use devices working in three different bands (visible, infrared and microwave) mounted on a single platform. Such detection systems can track detected target and visualize intrusion on operator interface simultaneously. Furthermore, these systems take advantage of two detection technologies: radar and camera unit.

Radar-camera systems are third generation perimeter protection systems [48]. These units are at early stage of development. Complete systems of observation platform are not available yet on the market.

Mobile radar-camera unit was developed as a part of above water/ground surveillance system [9]. This solution allowed us to extend possibilities of threats detection and identification by the system with ensured mobility of test unit. Thanks to this solution it is possible to carry out tests and set up the optimal placement of radar-camera units on protected area.

Occurrence of terrorist attack in sea area of the port is also possible. The risk level of such attacks are comparable to attacks from the ground. Attempts of breaking to the port area can be realized by underwater vessels (manned and unmanned miniature submarines) or small surface vessels such as small boats, canoes, windsurfing boards, rafts or floating explosive devices and divers equipped with weapons or explosives.

Table 1: $\quad$ Parameters of a multispectral surveillance system.

\begin{tabular}{|l|l|}
\hline \multicolumn{2}{|c|}{ Image resolution } \\
\hline Vis camera & $1024(\mathrm{H}) \times 768(\mathrm{~V})$ pixels \\
\hline IR camera & $320(\mathrm{H}) \times 240(\mathrm{~V})$ pixels \\
\hline \multicolumn{2}{|c|}{ Field of view (FOV) } \\
\hline Vis camera & $2^{\circ}$ to $45^{\circ}$ \\
\hline IR camera & $14^{\circ}$ \\
\hline \multicolumn{2}{|c|}{ Radar detection range } \\
\hline \multicolumn{2}{|c|}{ Radar resolution } \\
\hline - min. detection distance & $5 \mathrm{~m}$ \\
\hline - depth resolution & $0.5 \mathrm{~m}$ \\
\hline - separate target detection & $2.5 \mathrm{~m}$ \\
\hline
\end{tabular}


Underwater object detection is possible, by passive measurement systems, especially by using correctly positioned magnetic or hydroacoustic barriers, sonars and passive measurement modules. Barriers should be capable of detecting, identifying, locating and tracking objects detected in the observation area. Additionally, to increase detection reliability of floating objects in the harbor area, as a part of system, passive measurement modules should be distributed. These modules can be equipped with sensors measuring physical fields of ships.

To ensure proper crisis management actions, a system demonstrator which uses innovative security and monitoring technologies, for the protection of seaports areas is proposed. Software integration of underwater and ground/above water surveillance subsystems enables the building of a demonstrator of a multisensor security system $[10,11]$. An integrated demonstrator ensures complex threat detection and identification in seaport areas.

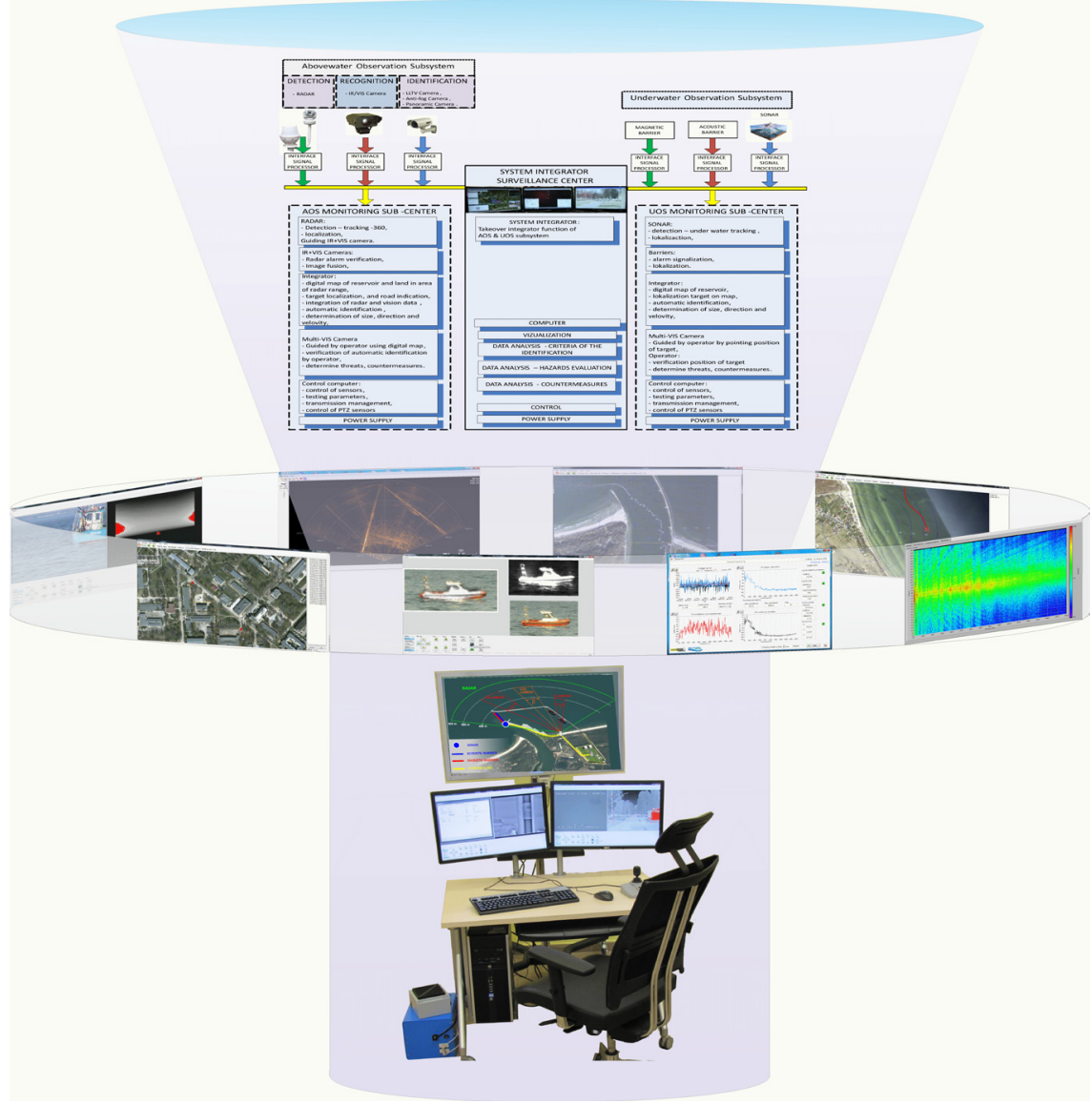

Figure 2: Command and control center demonstrator. 
Software gives the possibility of carrying out fusion of data from different sensors [12]. This allows us to provide the highest performance of threat detection and identification. To achieve full capabilities of the system, the demonstrator of the Command and Control Center was developed (Fig. 2). The center allows the operator to access all functionalities and optimize the amount of data collected by system sensors, although the operator cannot disable selected functionalities necessary for proper system operation. The demonstrator has the ability to work as a manager unit of various subsystems, or as a superior management unit of an integrated system.

\section{Laser photography system}

The laser photography system is an advanced experimental system for acquisition and distribution of spatial information. Its key element is a laser photography device (LPD) using active illumination for scene observation [1315]. The system also includes other information units such as VIS, IR, NIR cameras, and millimetre wave radar. The studies are characterized with the individual hardware components and as the system.

The presented system draws attention not only on the properties offered by the LPD but also the possibility of synergistic effects of the entire system through the fusion of data from different sensor units.

Laser Photography Device LPD can work independently. However, to extend its functionalities and show capabilities compared to other vision surveillance devices, Laser Photography System (LPS) was developed (Fig. 3). The main element of the system is the LPD, but it also contains positioning platform on which LPD is mounted, radar to identify targets and video cameras operating in VIS-NIR-IR spectrum.

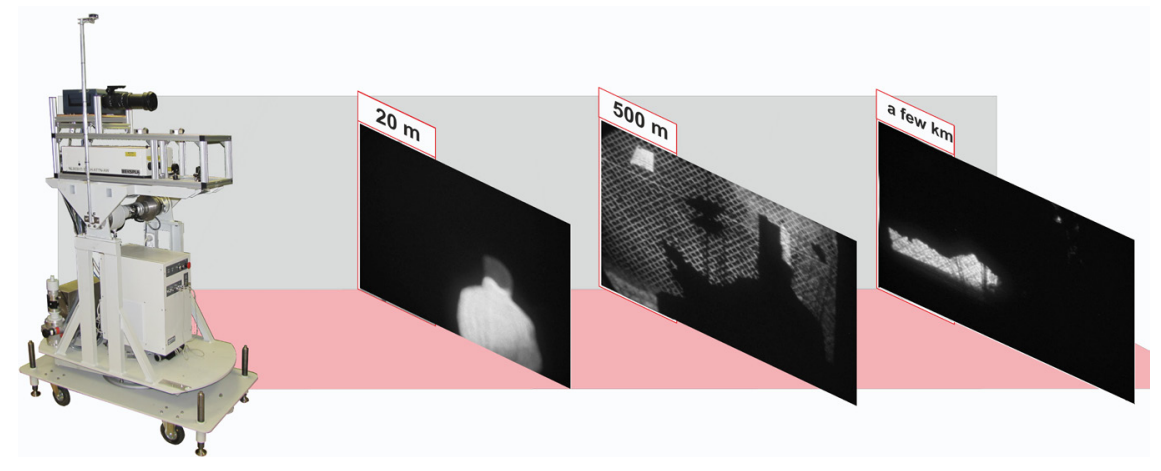

Figure 3: $\quad$ Laser photography system (LPS).

During the system development the authors assumed that the key element of the system is a device using time-spatial framing method [16]. Based on this assumption an observation measurement system was developed. All additional components in the system work for the LPD or are complementary to its functionalities. 
Table 2: $\quad$ Imaging parameters of LPS.

\begin{tabular}{|c|c|}
\hline \multicolumn{2}{|c|}{ Image resolution } \\
\hline \multicolumn{2}{|c|}{$1360(\mathrm{H}) \times 1024(\mathrm{~V})$ pixels } \\
\hline \multicolumn{2}{|c|}{ MTF resolution } \\
\hline \multicolumn{2}{|c|}{$601 \mathrm{p} / \mathrm{mm}$} \\
\hline \multicolumn{2}{|c|}{ Field of view (FOV) } \\
\hline - for $105 \mathrm{~mm}$ optics & $11,04^{\circ}$ \\
\hline - for $500 \mathrm{~mm}$ optics & $2,46^{\circ}$ \\
\hline - for $2800 \mathrm{~mm}$ optics & $0,38^{\circ}$ \\
\hline Bit resolution & 14 - bits \\
\hline \multicolumn{2}{|c|}{$\begin{array}{l}\text { Spatial resolution } \\
\end{array}$} \\
\hline - min. observation distance & $16 \mathrm{~m}$ for $532 \mathrm{~nm}$ illuminator \\
\hline & $0 \mathrm{~m}$ for $850 / 905 \mathrm{~nm}$ illuminator \\
\hline - min. observation depth & $1.2 \mathrm{~m}$ \\
\hline - min. distance increment & $0.15 \mathrm{~m}$ \\
\hline - min. depth increment & $0.15 \mathrm{~m}$ \\
\hline
\end{tabular}

In the developed system, the following modules are used as additional sensors:

- VIS-NIR-IR cameras,

- Millimeter wave radar,

- $\quad$ ERT- sensors (Earth Relation Tracking).

Comparing the LPD with other vision devices used in video surveillance systems, the LPS uses cameras working in three spectral bands VIS, NIR and IR [17]. The proposed solution, by using an active laser illumination of scene offers bigger range of observation (the solution is resistant to time of day, presence of light sources or lighting conditions), enables interpretation of the video material with aspects offered by traditional monitoring systems.

\section{THz-VIS imaging system}

A growing interest in terahertz technologyfinds support in a large number of applications. One of the most interesting applications of terahertz waves is imaging [18]. The terahertz range of electromagnetic radiation has large potential in the field of hidden objects detection because it is not harmful to humans. However, the main difficulty in the $\mathrm{THz}$ imaging systems is low image quality due to low sensitivity and a small number of pixels in detecting modules of cameras.

Considering the fact that even $\mathrm{THz}$ images with low pixel resolution still provide valuable information, it is justified to combine them with the highresolution images from a visible camera [19].

For our studies, we chose the TS4 camera from ThruVision Systems Ltd., which seems to have quite good performance and image quality. The camera 
Table 3: $\quad$ Parameters of a THz-VIS imaging system.

\begin{tabular}{|l|l|}
\hline \multicolumn{2}{|c|}{ Image resolution } \\
\hline Vis camera & $\begin{array}{l}3872(\mathrm{H}) \times 2592(\mathrm{~V}) \text { 10fps } \\
550(\mathrm{H}) \times 600(\mathrm{~V}) \quad 30 \mathrm{fps} \\
+ \text { motin tracking }\end{array}$ \\
\hline THz camera & $124(\mathrm{H}) \times 271(\mathrm{~V})$ pixels \\
\hline \multicolumn{2}{|c|}{ Field of view (FOV) } \\
\hline Vis Camera & $87^{\circ}$ \\
\hline \multicolumn{2}{|c|}{ Object detection range } \\
\hline
\end{tabular}

detects natural human radiation at the frequency $0.25 \mathrm{THz}$ thanks to the fact that clothing transmits well in this range. A heterodyne detection method based on a GaAs Schottky mixer combined with a local oscillator. The number of pixels is 124 x 271. TS4 can detect objects hidden under clothing (bombs, guns, knives, wallets, belts, etc.) due to their different temperature and emissivity than human body. The main idea of our current efforts refers to improvement of the image recorded for long distances $(>5 \mathrm{~m})$ and small sizes of hidden on a person objects.

Pure passive systems working in the millimetre wave region can detect hidden on persons objects thanks to difference in naturally emmited power between human body and the objects.

The aim of operation of $\mathrm{THz}$ based multispectral imaging system is to detect and visualize objects hidden under clothing. Detection of potentially dangerous objects is important, but without proper visualization, can be difficult to utilize. Security applications very often require a person to operate a system, thus the fused image is intended for presentation to a human observer for easier and enhanced interpretation.

$\mathrm{THz}$ images are more difficult to assimilate for a human eye than visual images because they present a reality invisible for humans. Visible images are natural for human vision. Our studies show that the fused image should contain information mostly from the visible image with superimposed elements from the THz image showing a detected object.

An imaging system is usually composed of various subsystems. Many of the imaging systems use imaging devices working in various spectral ranges. The presented system draws attention not only on the properties offered by a single camera but also on the potential of synergetic effects of the system by using the fusion of data registered by different sensor units. Our system consists of the $\mathrm{THz}$ camera and the VIS camera mounted on the mobile platform. The multispectral system with an example of fused images are presented in Figure 4. 


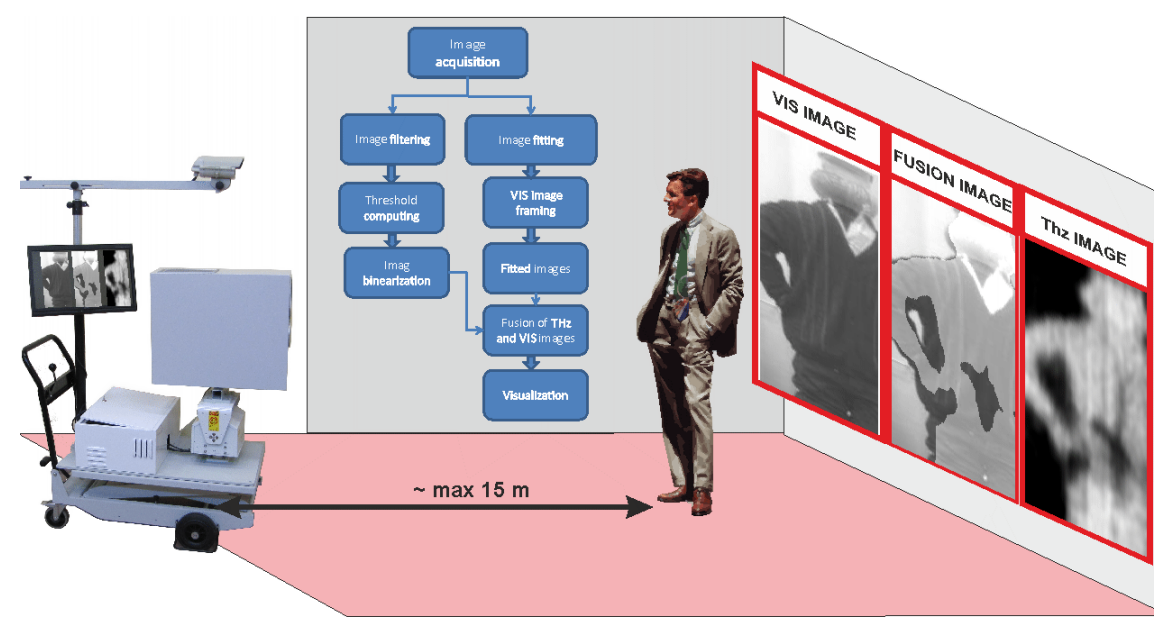

Figure 4: $\quad$ Multispectral imaging system based on THz and VIS cameras.

\section{Conclusion}

Multispectral detection is a basis of all security systems developed in IOE MUT which allows significant increase of security of protected objects. Because of systems mobility they can be tested in field conditions to optimize deployment and performance of such system. Furthermore, they can be used for protection of objects operating periodically like mobile military bases or command centers. Processing and fusion methods of data from devices working in multiple spectral bands provide greater probability of threats detection and accurate identification. These systems are designed to fill gaps in currently used security systems and to ensure maximal protection of critical infrastructure objects.

\section{References}

[1] Zyczkowski M., Szustakowski M., Ciurapinski W., Karol M., Markowski P., Integrated radar-camera security system: Range test, Proceedings of SPIE 8361, art. no. 83611G (2012)

[2] Dulski R., Szustakowski M., Kastek M., Ciurapinski W., Trzaskawka P., Zyczkowski M., Infrared uncooled cameras used in multi-sensor systems for perimeter protection, Proc. of SPIE, 7834, 783416 (2010)

[3] Dulski R., Milewski S., Kastek M., Trzaskawka P., Szustakowski M., Ciurapinski W., Zyczkowski M., Detection of small surface vessels in near, medium and far infrared spectral bands, Proceedings of SPIE 8185 , art. no. 81850U (2011)

[4] Zyczkowski M. , Szustakowski M., Ciurapinski W., Palka N., Kastek M., Integrated optoelectronics security system for critical infrastructure protection, Przeglad Elektrotechniczny 86 (10), 157 (2010) 
[5] Zyczkowski M., Arciuch A., Ciurapinski W., Wielosensorowy system ochrony naziemnej obiektów strategicznych, WKŁ ISBN 978-83-2061746-7, 2009

[6] Zyczkowski M., Szustakowski M., Kastek M., Ciurapinski W., Sosnowski T., Module multisensor system for strategic objects protection, WITPRESS ISBN 978-1-84564-184-9, 2009

[7] Zyczkowski M., Szustakowski M., Ciurapinski W., Dulski R., Kastek M., Trzaskawka P., Integrated mobile radar-camera system $\mathrm{n}$ airport perimeter security, Proceedings of SPIE 8184 , art. no. 818406 (2011)

[8] Zyczkowski M., Palka N., Trzcinski T., Dulski R., Kastek M., Trzaskawka P., Integrated radar-camera security system - Experimental results, Proceedings of SPIE 8021 , art. no. 80211U (2011)

[9] Szustakowski M., Ciurapinski W., Zyczkowski M., Palka N., Kastek M., Dulski R., Bieszczad G., Sosnowski T., Data fusion concept in multispectral system for perimeter protection of stationary and moving objects, Proceedings of SPIE 7481, 74810D (2009)

[10] Kastek M., Dulski R., Zyczkowski M., Szustakowski M., Trzaskawka P., Ciurapinski W., Grelowska G., Listewnik K., Multisensor system for the protection of critical infrastructure of a seaport, Proceedings of SPIE 8388 , art. no. 83880M (2012)

[11] Kastek M., Dulski R., Zyczkowski M., Szustakowski M., Ciurapinski W., Firmanty K., N. Pałka, Bieszczad G., Multisensor systems for security of critical infrastructures - Concept, data fusion, and experimental results, Proceedings of SPIE 8193 , art. no. 81933X (2011)

[12] Dulski R., Kastek M., Trzaskawka P., Piatkowski T., Szustakowski M., Zyczkowski M., Concept of data processing in multi-sensor system for perimeter protection, Proceedings of SPIE 8019, art. no. 80190X (2011)

[13] Piszczek M., K. Rutyna, Szustakowski M., Applications of the laserphotography, Elektronika - Konstrukcje, Technologie, Zastosowania nr $10 / 2010$

[14] Piszczek M., Kowalski M., Szustakowski M., Rutyna K., Ludwikowski K., The role of a laser photography device illuminator in acquisition of spatial information, Acta Physica Polonica A122, 862 (2012)

[15] Piszczek M., Metadata in a LPS, Acta Physica Polonica A122, 858 (2012)

[16] Piszczek M., New possibilities of the anti-terrorist protection systems with using augmented reality, Przegląd Elektrotechniczny 9a, 45 (2011)

[17] Piszczek M., M. Zarzycki Szustakowski M., Elements of augmented reality for vision systems, Acta Physica Polonica A122, 950 (2012)

[18] Kowalski M., Piszczek M., Palka N., Szustakowski M., Processing of THz images acquired by a passive camera, Phot. Lett. Poland 4, 3 (2012)

[19] Kowalski M., Piszczek M., Palka N., Szustakowski M., Improvement of passive THz camera images, Proceedings of SPIE, 8544 , art. no. $85440 \mathrm{~N}-1$ (2012) 